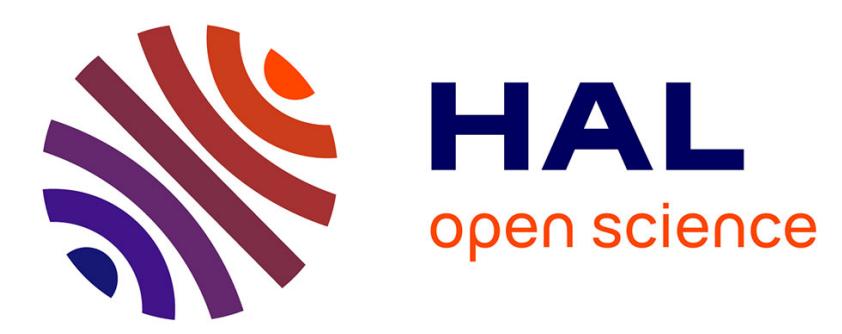

\title{
Towards a continuous approach of functional-structural plant growth
}

\author{
Zhongping Li, Vincent Le Chevalier, Paul-Henry Cournède
}

\section{To cite this version:}

Zhongping Li, Vincent Le Chevalier, Paul-Henry Cournède. Towards a continuous approach of functional-structural plant growth. Third International Symposium on Plant Growth Modeling, Simulation, Visualization and Applications (PMA09), Nov 2009, Beijing, China. pp.334-340, 10.1109/PMA.2009.69 . hal-00529351

\section{HAL Id: hal-00529351 \\ https://hal.science/hal-00529351}

Submitted on 25 Oct 2010

HAL is a multi-disciplinary open access archive for the deposit and dissemination of scientific research documents, whether they are published or not. The documents may come from teaching and research institutions in France or abroad, or from public or private research centers.
L'archive ouverte pluridisciplinaire HAL, est destinée au dépôt et à la diffusion de documents scientifiques de niveau recherche, publiés ou non, émanant des établissements d'enseignement et de recherche français ou étrangers, des laboratoires publics ou privés. 


\title{
Towards a continuous approach of functional-structural plant growth
}

\author{
Zhongping Li*†‡, Vincent Le Chevalier* ${ }^{* \ddagger}$, Paul-Henry Cournède ${ }^{* \ddagger}$ \\ *Ecole Centrale Paris, Laboratory of Applied Maths and Systems - Châtenay-Malabry, France \\ zhongping.li@ecp.fr \\ ${ }^{\dagger} U M R$ G-EAU, Cemagref, Montpellier, France \\ ${ }^{\ddagger}$ INRIA Saclay Ille-de-France, EPI Digiplante - Orsay, France
}

\begin{abstract}
We propose in this article a continuous approach to model functional-structural plant growth based on the discrete GreenLab model. The continuous dynamics is driven by a system of differential equations with respect to calendar time, with a continuous mechanism of senescence introducing delay terms. A numerical scheme for solving the system is studied, and applied to sugar beet to compare different approximation methods including the classical discrete model. With a higher precision, the simulation based on the continuous approach reveal significant differences with the discrete model. Moreover, an approximation of the continuous model is derived with a daily time step, which makes it suitable for agronomy applications.
\end{abstract}

\section{Introduction}

Functional-structural plant models (FSPM) combine plant architecture description with ecophysiological processes ([1]). In models of this type, organogenesis is traditionally computed as a discrete process, based on parallel rewriting formal grammars ([2], [3] for seminal works or [4] more recently). In particular, the previous versions of the GreenLab growth model have chosen the growth cycle (that is to say the thermal time between the appearance of successive growth units) as the time unit to formulate the growth dynamics in a discrete recurrent system ([5], [6]).

This approach of discretization may involve some problems, which we endeavour to overcome by proposing a continuous model of plant growth derived from the GreenLab model.

First, physiological processes, that govern plant morphology, are driven by environmental factors (temperature, photosynthetically active radiation, etc) which continuously vary with calendar time. Typically, the environmental data are collected daily, and we would also expect to have daily outputs of the growth model ([7], [8]). Growth cycle does not correspond to a constant time step with respect to calendar time and may vary from several days to a year for different plants. Thus, discrete models based on the architectural growth cycle may prove to be inconvenient and inaccurate in landscape and crop applications for which interactions with a changing environment have to be taken into account ([9]).

Inaccuracies are also introduced by modeling the interactions between plant morphology and physiological processes in a discrete way. For example, in previous versions of GreenLab, the biomass produced by photosynthesis during a cycle is calculated according to the foliar surface area at the end of the previous cycle, and blades stop functioning brutally at the end of their functioning time.

In Section 2, we describe a continuous adaptation of the GreenLab model. We formulate biomass variation with respect to calendar time in the form of integral and differential equations, first for the total foliar mass of the plant, then for each organ. Blade senescence is also modeled in a continuous way, which introduces a delay term in the differential equations.

Then we propose a numerical scheme to solve the equations for accumulated foliar mass, by dividing the time axis into intervals, to specify the presence of the two major factors (photosynthesis and blade senescence), and also to adapt the numerical resolution for delay differential equations.

At last, we present simulation results on different test cases for sugar beet, in order to compare various approximation schemes for the continuous model and the discrete model.

\section{A continuous model of plant growth}

\subsection{Calendar time versus thermal time}

The choice of time unit is crucial to describe the interaction of plant growth with the environment. The variations of the environment are best described as functions of calendar time, while plant development and organ expansion are closely related to thermal time ([10]), defined as:

$$
\tau(t):=\int_{0}^{t} \max \left(0, T(s)-T_{b}\right) d s
$$

with $T_{b}$ the base temperature.

Growth cycles are defined by the appearance of new architectural growth units ([11]). A growth unit is composed 
of a determined number of different types of organs that we suppose given by an organogenesis model not described here (see for example [6]). Let $N_{o}(k)$ denote the number of organs of type $o(o=b, i, p, r$ for blade, internode, petiole, and root respectively) generated at the $k$-th cycle. Generally for crops, the thermal time elapsing between successive appearances of phytomers can be considered as constant and is called phyllochron. It is denoted by $\gamma$ and is regarded as the growth cycle in GreenLab. In the following, we consider that organogenesis increments occur at integer multiples of $\gamma$. The thermal expansion time of each type of organ is assumed to be constant, denoted by $\tau_{o}$.

\subsection{Production}

We consider that $\tau(0)=0$ at plant initiation, and thus at plant emergence, $\tau(t)=\gamma$. After emergence (the initial period before emergence is treated in section 2.5), we assume that the cumulated dry matter $Q(t)$ produced by photosynthesis till time $t\left(t \in\left[\tau^{-1}(\gamma), \infty[)\right.\right.$ is given by the differential equation:

$$
\frac{d Q(t)}{d t}=\frac{d E(t)}{d t} \alpha(1-\exp (-\beta S(t)))
$$

with $E(t)$ a function of the environment. Here we take $E(t)=P A R(t)$ where $P A R(t)$ denotes the incident photosynthetically active radiation accumulated since plant emergence till $t$, and is assumed to equal 0.48 times the global incident radiation ([12]). $S(t)$ is the total leaf surface area of the plant at time $t$, and $\alpha, \beta$ are empirical coefficients, as detailed in Section 4. This equation derives from the discrete model ([13]).

Blades are also assumed to have a constant specific blade mass $e$, i.e. $S(t)=\frac{Q_{b}(t)}{e}, Q_{b}$ denoting the accumulated active foliar mass.

\subsection{Allocation}

At time $t, \frac{d Q(t)}{d t}$ is distributed to each organ proportionally to its sink strength ([14]). We define sink strength functions as continuous versions of those defined in [14]. The sink strength of an organ of type $o$, is a function of its thermal age $u$, given by:

$$
\mathrm{P}_{o}(u)=p_{o} f_{a_{o}, b_{o}}(u)
$$

for $0 \leq u \leq \tau_{o}$, and $\mathrm{P}_{o}(u)=0$ otherwise. $p_{o}$ is organ base sink strength, and $f_{a_{o}, b_{o}}$ is the normalized beta function defined as:

$$
f_{a_{o}, b_{o}}(u)=\frac{1}{M_{o}}\left(\frac{u}{\tau_{o}}\right)^{a_{o}-1}\left(1-\frac{u}{\tau_{o}}\right)^{b_{o}-1}
$$

with $M_{o}:=\sup _{u \in\left[0, \tau_{o}\right]}\left(\frac{u}{\tau_{o}}\right)^{a_{o}-1}\left(1-\frac{u}{\tau_{o}}\right)^{b_{o}-1}$.
Therefore, when plant has thermal age $u(u=0$ at plant's initiation), the demand of all the organs of type $o$ is the sum of all their sink strengths:

$$
P_{o}(u)=\sum_{k \in \mathbb{N}} N_{o}(k) \mathrm{P}_{o}(u-k \gamma)
$$

Finally, we denote:

$$
D(u):=\sum_{o=b, i, p, r} P_{o}(u)
$$

the total demand of the plant at thermal time $u$.

If $q_{o, k}$ denotes the accumulated biomass allocated to an organ of type $o$ appeared at cycle $k$ (either from seed biomass or from photosynthesis), then we have for time $t \in\left[\tau^{-1}(\gamma), \infty[:\right.$

$$
\frac{d q_{o, k}(t)}{d t}=\frac{\mathrm{P}_{o}(\tau(t)-k \gamma)}{D(\tau(t))} \frac{d Q(t)}{d t}
$$

\subsection{A new formulation of plant senescence adapted to the continuous model}

Contrary to the discrete GreenLab model that considers that organs die instanteneously at the end of their lifespan, we introduce a new model of progressive senescence, describing more precisely the gradual death of tissues. Such idea is actually considered in other so-called discrete models ([15], [16]). Here we assume that all organ tissues have the same longevity $\tau_{o}^{s e n}$, corresponding to the period between its initiation and beginning of senescence ([17]).

We consider in the following that internodes and root do not get senescent $\left(\tau_{i}^{\text {sen }}=\tau_{r}^{\text {sen }}=\infty\right)$. Therefore senescence only concerns leaves, and we take the same senescence time for both blades and petioles $\left(\tau_{b}^{s e n}=\tau_{p}^{s e n}=\tau^{s e n}\right)$. This case corresponds to Sugar Beet growth ([13]), that is considered in our numerical tests.

If $Q_{o, k}$ is the accumulated mass of the organ $o$ initiated at cycle $k$, according to the senescence mechanism described we get for $o=b, p$ and $t \in\left[\tau^{-1}\left(\tau^{\text {sen }}\right),+\infty[\right.$ :

$$
\frac{d Q_{o, k}(t)}{d t}=\frac{d q_{o, k}}{d t}(t)-\frac{d q_{o, k}}{d t}(\eta(t))
$$

with $\eta(t):=\tau^{-1}\left(\tau(t)-\tau^{s e n}\right)$.

And when $o=b, p$ and $t \in\left[0, \tau^{-1}\left(\tau^{\text {sen }}\right)[\right.$, or when $o=$ $i, r$ and $t \in[0,+\infty[$ :

$$
\frac{d Q_{o, k}(t)}{d t}=\frac{d q_{o, k}}{d t}(t)
$$

From (1)-(5), we can see that, after plant's emergence, the dynamics of $Q_{b}$ drives that of the whole system. Thus in what follows, we concentrate on the variations of $Q_{b}$. 


\subsection{The initial phase}

During cycle 0 , that is to say from plant's initiation till its emergence (visual appearance of the first phytomer), we assume that the seed distributes its mass uniformly in thermal time to organs of the first growth unit, therefore for $t \in\left[0, \tau^{-1}(\gamma)[:\right.$

$$
Q_{b}(t)=\int_{0}^{\tau(t)} \frac{Q^{\text {seed }}}{\gamma} \frac{P_{b}(u)}{D(u)} d u
$$

$Q^{\text {seed }}$ denoting the seed mass.

\subsection{A delay system}

By summarizing the above, we get the complete evolution of accumulated foliar mass. Namely, after the initial phase formulated by (6), foliar mass has a pure growth phase till blades start to fade: for $t \in\left[\tau^{-1}(\gamma), \tau^{-1}\left(\tau^{\text {sen }}\right)[\right.$,

$$
\frac{d Q_{b}(t)}{d t}=F\left(t, Q_{b}(t)\right)
$$

where:

$$
F\left(t, Q_{b}(t)\right):=\frac{\bar{P}_{b}}{\bar{D}}(t) \frac{d P A R(t)}{d t} \alpha\left(1-\exp \left(-\frac{\beta}{e} Q_{b}(t)\right)\right)
$$

$t \in\left[\tau^{-1}(\gamma), \infty\left[\right.\right.$. With $\bar{P}_{o}(t):=P_{o}(\tau(t)), o=b, i, p, r$, and $\bar{D}(t):=D(\tau(t))$.

Then senescence should be taken into account. Its mechanism has been presented in section 2.3. As a delay effect of cycle 0 , we have for $t \in\left[\tau^{-1}\left(\tau^{\text {sen }}\right), \tau^{-1}\left(\tau^{\text {sen }}+\gamma\right)[\right.$ :

$$
\frac{d Q_{b}(t)}{d t}=F\left(t, Q_{b}(t)\right)-\frac{Q^{\text {seed }}}{\gamma} \frac{\bar{P}_{b}}{\bar{D}}(\eta(t))
$$

And at last for $t \in\left[\tau^{-1}\left(\tau^{\text {sen }}+\gamma\right),+\infty[\right.$ :

$$
\frac{d Q_{b}(t)}{d t}=F\left(t, Q_{b}(t)\right)-F\left(\eta(t), Q_{b}(\eta(t))\right)
$$

We remark in particular that (8) and (9) are delay differential equations, with (6) and (7) as initial functions (see [18]).

\section{Numerical approximation}

\subsection{General scheme}

Now we show briefly how to solve these equations numerically, in particular by adapting the delay term .

The time axis is first divided into intervals on which are defined equation (6)-(9). Then, as explained below, the last phase $\left[\tau^{-1}\left(\tau^{\text {sen }}+\gamma\right),+\infty[\right.$ is divided again into intervals corresponding to the the thermal time of senescence $\tau^{\text {sen }}$, to adapt the numerical solution of (9).

All the numerical schemes are one-step methods. We choose a constant time step $h$ for all these equations, except for the last step of each interval. After a maximal number of steps of length $h$, we take the remaining time as the last step of the interval, to cover it completely without overlap with the next interval.

The integral (6) can be easily approximated, for example by the trapezium rule, and we can apply Runge-Kutta methods to solve the relevant ordinary differential equations (7).

As for the phase after emergence, we propose to solve the delay differential equations with the method of steps (see [18]). For this purpose, we divide the time axis into intervals $\left[t_{k}, t_{k+1}[\right.$ :

$$
t_{k}:=\tau^{-1}\left(k \tau^{\text {sen }}\right), k=0,1, \ldots
$$

By interpolating the solutions of (6) and (7), we get the approximated value of $Q_{b}$ on the whole interval $\left[t_{0}, t_{1}[\right.$, then (8) and (9) can be written in the following form on on $\left[t_{1}, t_{2}[:\right.$ the form of:

$$
\frac{d Q_{b}(t)}{d t}=F\left(t, Q_{b}(t)\right)-G(t)
$$

It involves a function $G$ which has already been approximated. Then we apply Runge-Kutta to (10) on $\left[t_{1}, t_{2}[\right.$, we interpolate and we move to $\left[t_{2}, t_{3}[\right.$, etc.

As for the calculation at organ level, it is similar with that of $Q_{b}$ for $t \in\left[0, \tau^{-1}(\gamma)\left[\right.\right.$. For $t \in\left[\tau^{-1}(\gamma),+\infty[\right.$, it is in the form of:

$$
\frac{d Q_{o, k}(t)}{d t}=H\left(t, Q_{b}(t)\right)
$$

by using equations (1)-(5), so that $Q_{o, k}$ can be fully approximated once $Q_{b}$ is solved.

\subsection{The daily model}

As usually the available environmental data for temperature and $P A R$ are given daily, we specifically derive the numerical approximation of our continuous model for a daily time step, i.e. $h=1$ when solving (6)-(9). This particular scheme shall prove useful for agronomic applications. And we detail in this example case how environmental functions are approximated.

If $\bar{T}(i):=\int_{i}^{i+1} T(t) d t$ is the average temperature of day $i$, then we approximate $\tau(t)$ as:

$$
\tau(t) \approx \sum_{i=0}^{\mathbf{E}(t)} \bar{T}(i)+(t-\mathbf{E}(t)) \bar{T}(\mathbf{E}(t))
$$

$\mathbf{E}(t)$ denoting the integer part of $t$.

As for $P A R$, we will take for all $i \in \mathbb{N}$ :

$$
\frac{d P A R(t)}{d t} \approx \overline{P A R}(i), t \in[i, i+1[
$$

with $\overline{P A R}(i):=P A R(i+1)-P A R(i)$.

We remark that methods requiring intermediate value within a step like Simpson for integral approximation or 
fourth-order Runge-Kutta for differential equation solution are not necessary, since temperature and $d P A R$ are approximated constantly within a step.

\section{A test case on sugar beet}

Simulations are carried out based on the GreenLab model of sugar beet growth [13], in order to compare the impact of different methods when calculating biomass production and accumulated organ masses. For different environmental conditions, we simulate biomass production, foliar mass and root mass with three methods: first the discrete model adapted to take into account the gradual senescence mechanism mentioned in Section 2.4, and then the continuous model by solving numerically the dynamic equations on one hand with Euler's method and and on the other hand with Second-order Runge-Kutta method (Heun's method).

For organogenesis, each growth unit of Sugar Beet contains a blade and a petiole, thus we have in (2) $N_{b}(k)=$ $N_{p}(k)=1$ for all $k$ and $N_{r}(0)=1, N_{r}(k)=0$ for $k>0$. And $T_{b}=0^{\circ} \mathrm{C}$.

We simulate for 83 growth cycles in all simulations. Table 1 lists values of all constant parameters. With $\mu$ the empirical coefficient related to the radiation use efficiency, $k$ the Beer-Lambert extinction coefficient and $S_{p}$ the empirical coefficient corresponding to a characteristic surface, that specify the parameters in equation (1) as:

$$
\alpha:=\mu S_{p}, \beta:=\frac{k}{S_{p}}
$$

\subsection{Numerical studies}

In this section, we highlight the comparison among the three approximation methods. We consider a constant temperature $\bar{T} \equiv 17^{\circ} \mathrm{C}$ and $\gamma=34^{\circ} \mathrm{C}$.days, therefore $h=2$ corresponds to a growth cycle. We denote the constant $P A R$ received at each growth cycle by $\overline{P A R}_{G C}$ for the discrete model. If $\overline{P A R}_{d a y}=\frac{1}{2} \overline{P A R}_{G C}$, the discrete model and the numerical discretization of the continuous model have the same time step and environmental conditions. Three simulations are shown in Figure 1.-3., by varying the $\overline{P A R}_{G C}$.

For the convenience of description, we refer to the early phase of plant growth as the increasing phase when mass production increases and we refer to the late growth phase as the decreasing phase. Finally, the saturated phase corresponds to the intermediate phase when plant production saturates. The analysis of Figure 1.-3. reveals some interesting points which are discussed below.

1) For the cumulated biomass production $Q(t)$ (corresponding to the integral of the production curve in Figures 1.-3.) there is an important gap between RK2 and Euler in the increasing phase for all three
Table 1. Parameters for discrete and continuous simulations of sugarbeet growth ([13])

\begin{tabular}{ccc}
\hline Parameter & Value & Unit \\
\hline$\mu$ & 16.8 & $g / M J$ \\
$k$ & 0.7 & - \\
$S_{p}$ & 0.023 & $m^{2}$ \\
$e$ & 0.0083 & $g / \mathrm{cm}^{2}$ \\
$Q^{\text {seed }}$ & 0.003 & $g$ \\
$\gamma$ & 34 & ${ }^{\circ}$ C.days \\
$p_{b}$ & 1 & - \\
$p_{p}$ & 0.842 & - \\
$p_{r}$ & 400 & - \\
$a_{b}$ & 5.4 & - \\
$b_{b}$ & 5 & - \\
$a_{p}$ & 3.96 & - \\
$b_{p}$ & 5 & - \\
$a_{r}$ & 6 & - \\
$b_{r}$ & 3.7 & ${ }^{\circ}$ C.days \\
$\tau_{b}$ & 680 & ${ }^{\circ}$ C.days \\
$\tau_{p}$ & 680 & ${ }^{\circ}$ C.days \\
$\tau_{r}$ & 4080 &
\end{tabular}

simulations. Recalling that when solving (1) in the form of:

$$
\frac{d Q(t)}{d t}=f(t, Q(t))
$$

RK2 yields:

$$
Q_{t_{n+1}}^{R K}=Q_{t_{n}}^{R K}+\frac{k_{1}+k_{2}}{2} h
$$

with $k_{1}:=f\left(t_{n}, Q_{t_{n}}\right), k_{2}:=f\left(t_{n}+h, Q_{t_{n}}+k_{1} h\right)$. While Euler yields:

$$
Q_{t_{n+1}}^{E}=Q_{t_{n}}^{E}+k_{1} h
$$

Note that $q_{t_{n+1}}:=Q_{t_{n+1}}-Q_{t_{n}}$ for the mass production during time step $n+1$ in our tests, it is given by the production curve in Figures 1.-3. If $q_{t_{n}}^{R K}=q_{t_{n}}^{E}$, then:

$q_{t_{n+1}}^{R K}-q_{t_{n+1}}^{E}=\frac{h}{2}\left[f\left(t_{n}+h, y_{n}+k_{1} h\right)-f\left(t_{n}, y_{n}\right)\right]$

As $f$ increases in $t$ during the increasing phase, production by RK2 rises more quickly than that by Euler. Moreover, if the saturated phase is reached (see Figure 3. ), then in the decreasing phase RK2 declines more quickly than Euler, since $f$ decreases in $t$ during the decreasing phase. 


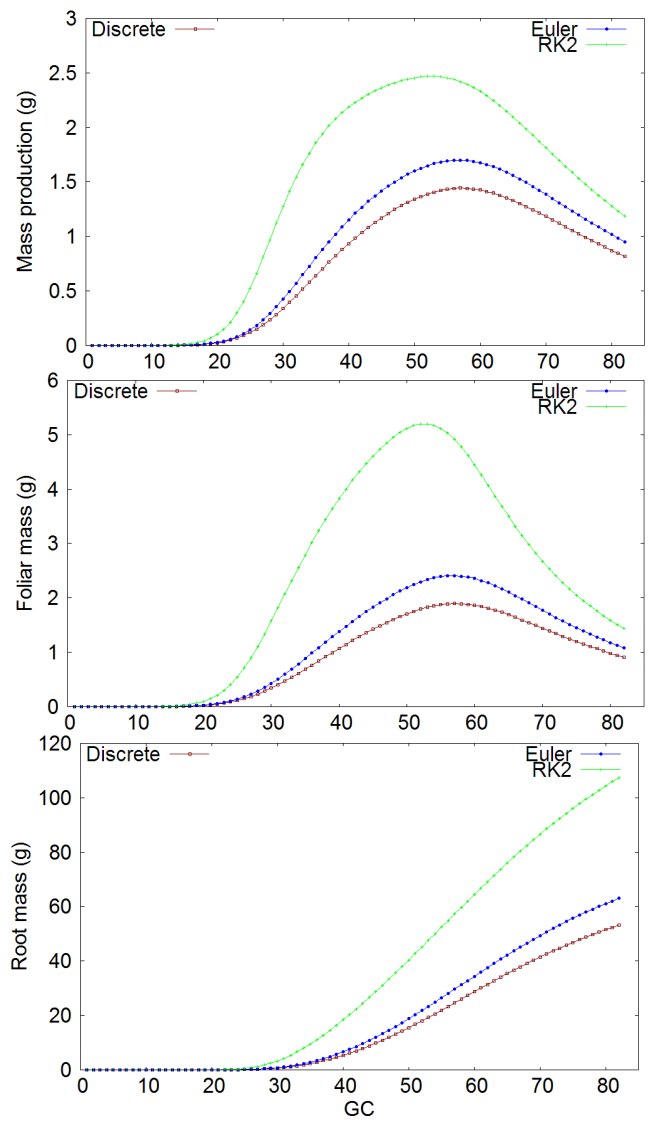

Figure 1. Biomass production, green leaf mass and root mass according to the growth cycle (= time step) for $\overline{P A R}_{G C} \equiv 7.5 \mathrm{MJ} / \mathrm{m}^{2}$.

2) Allocation favours blades at the early stages of plant growth, thus the gap between RK2 and Euler in the increasing phase has repercussions on foliar mass.

3) For root mass, the gap between RK2 and Euler is smaller for bigger $\overline{P A R}$. The reason is that in the late stages, the leaf surface area is important enough to yield an almost constant biomass production rate $\frac{d Q(t)}{d t}$ (given in the differential equation (11)), thus there is little difference between RK2 and Euler methods. Allocation to root is bigger in the late stages, therefore the root mass increases almost linearly during the saturated phase (see Figure 3.).

4) There is little difference between Euler and discrete method, while the differences are bigger with RK2. Actually, it comes out to be the same discretized formula of biomass production for both Euler's and discrete methods in these test cases. However, allocation is instantaneous in Euler's method, while for the discrete model, biomass partitioning is computed in the middle of each cycle.

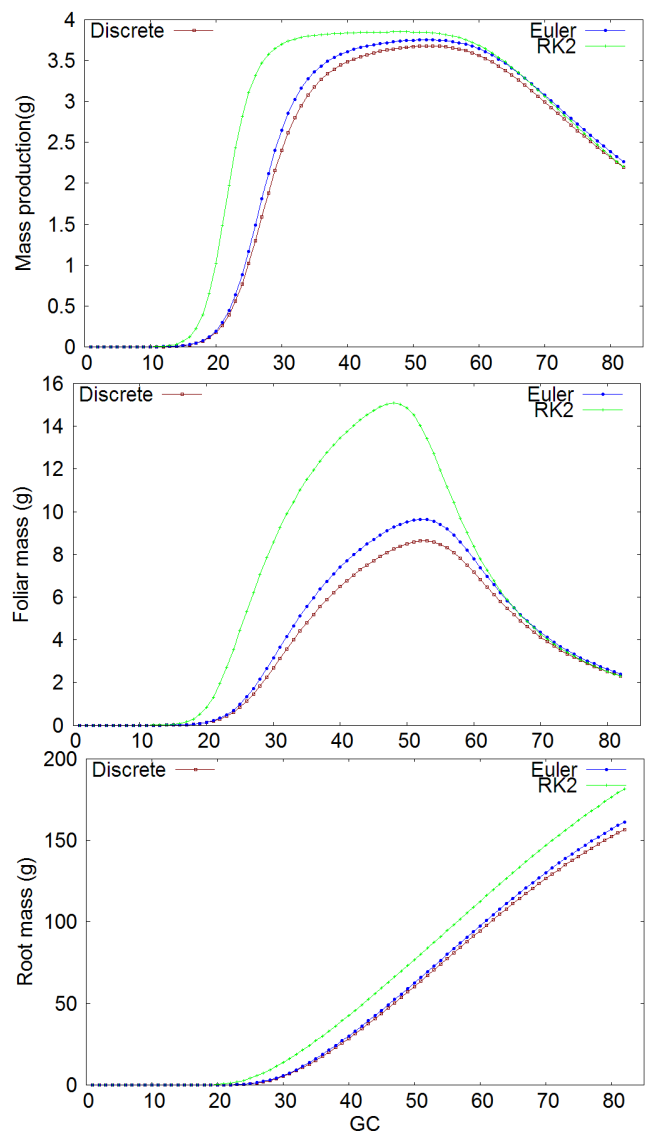

Figure 2. Biomass production, green leaf mass and root mass according to the growth cycle (= time step) for $\overline{P A R}_{G C} \equiv 10 \mathrm{MJ} / \mathrm{m}^{2}$.

\subsection{Simulation with real environmental data}

We used typical data observed in the north of France for $\bar{T}$ and $\overline{P A R}_{d a y}$ for a standard period of sugar beet growth (beginning of April to beginning of October). Simulation results are shown in Figure 4 for 83 growth cycles corresponding here to 170 days. We remark that in this realistic case, $\overline{P A R}_{d a y}$ is big enough for a saturated phase to appear.

\section{Discussion}

We proposed a continuous version of the GreenLab functional model to improve the model's precision and the description of the interactions with the environment. We also compared Euler's and RK2 schemes with the classical discrete GreenLab. The differences may be quite significant which leads to consider that the RK2 scheme shall be preferred.

The environmental data are usually available daily. For this reason, the daily approximation of the continuous model (detailed in Section 3.2) with RK2 should prove useful 

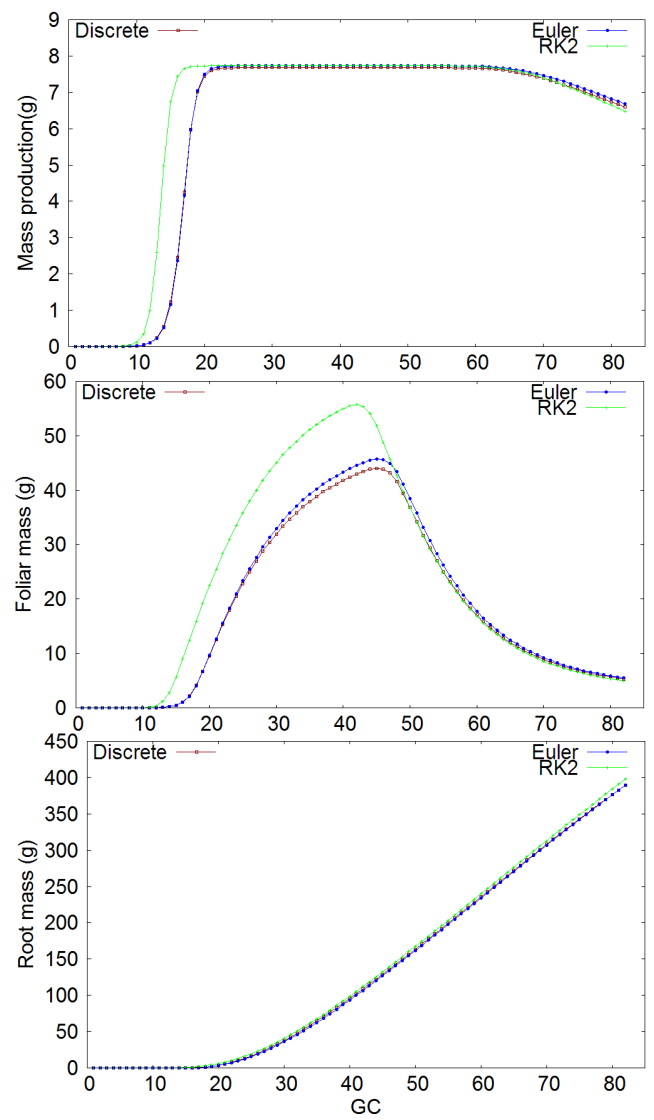

Figure 3. Biomass production, green leaf mass and root mass according to the growth cycle (= time step) for $\overline{P A R}_{G C} \equiv 20 M J / m^{2}$.

for most applications. For specific studies, for example in greenhouses when environmental data are collected at very fine scales and ecophysiological processes modelled with far more details, choosing a time step smaller than a day would be possible and more appropriate.

A key point in the development of predictive models is parameter stability. If the process studied is continuous, and the corresponding model is given by an inaccurate approximation, the parametric estimation from experimental data will also be inaccurate, leading to problems in parameter stability, for example parameter values that depend on the chosen time step. In this prospect, the proposed version of the GreenLab model should bring some key improvements over the discrete model.

Other more realistic senescence models may also be considered. Depending on the chosen model for senescence, some adaptations should be done to the delay system, without major difficulties.

Finally, we have only studied so far the continuous formulation of the functional part of GreenLab. In Section 2.1 , we have explicitly considered that organogenesis occurs
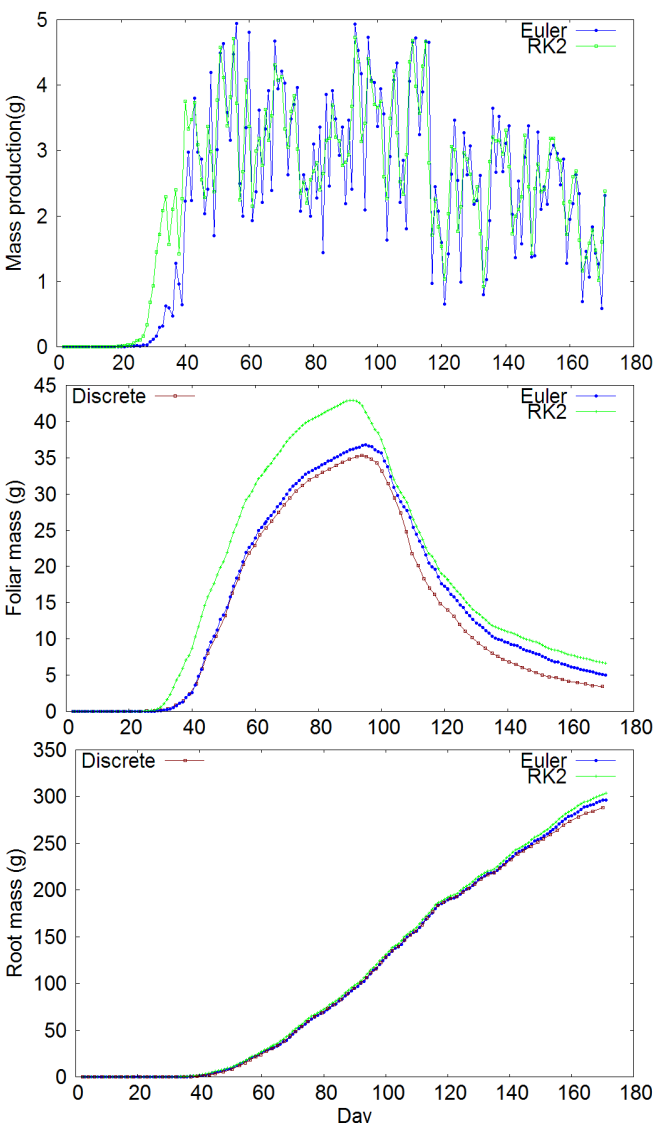

Figure 4. Biomass production, green leaf mass and root mass calculated daily for real $\bar{T}$ and $\overline{P A R}_{d a y}$.

at integer multiples of the phyllochron (or more generally of a constant thermal time increment). It clearly implies that the underlying organogenesis model is discrete. It would be interesting to integrate continuous organogenesis in this model with a continuous stochastic process of phytomer initiation.

\section{Acknowledgement}

This project is supported by the 3 Worlds project (ANR, France).

\section{References}

[1] J. Vos, L. Marcelis, and J. Evers, "Functional-structural plant modelling in crop production," in Functional-structural plant modelling in crop production, Wageningen, J. Vos, L. Marcelis, P. de Visser, P. Struik, and J. Evers, Eds., vol. Chapter 1. Springer, 2007.

[2] P. Prusinkiewicz and A. Lindenmayer, The Algorithmic Beauty of Plants. Springer-Verlag, New-York, 1990. 
[3] W. Kurth, Growth grammar interpreter GROGRA 2.4: A software tool for the 3-dimensional interpretation of stochastic, sensitive growth grammars in the context of plant modelling. Introduction and Reference Manual. Berichte des Forschungszentrums Waldökosysteme der Universität Göttingen, Ser. B, Vol. 38, 1994.

[4] C. Loi and P.-H. Cournède, "Generating functions of stochastic L-systems and application to models of plant development," Discrete Mathematics and Theoretical Computer Science Proceedings, vol. AI, pp. 325-338, 2008.

[5] P. de Reffye, M. Goursat, J. Quadrat, and B. Hu, "The Dynamic Equations of the Tree Morphogenesis Greenlab Model,” INRIA, Tech. Rep. 4877, 2003.

[6] P.-H. Cournède, M.-Z. Kang, A. Mathieu, J.-F. Barczi, H.-P. Yan, B.-G. Hu, and P. de Reffye, "Structural Factorization of Plants to Compute their Functional and Architectural Growth," Simulation, vol. 82, no. 7, pp. 427-438, 2006.

[7] E. Heuvelink, "Re-interpretation of an experiment on the role of assimilate transport-resistance in partitioning in tomato," Annals of Botany, vol. 78, pp. 467-470, 1996.

[8] L. Marcelis, E. Heuvelink, and J. Goudriaan, "Modelling of biomass production and yield of horticultural crops: a review," Scientia Horticulturae, vol. 74, pp. 83-111, 1998.

[9] V. Le Chevalier, M. Jaeger, X. Mei, and P.-H. Cournède, "Simulation and visualisation of functional landscapes: effects of the water resource competition between plants," Journal of computer science and technology, vol. 22, no. 6, pp. 835-845, Nov. 2007.

[10] G. Mac Master and W. Wilhelm, "Growing degree-days: one equation, two interpretations," Agricultural and Forest Meteorology, vol. 87, no. 4, pp. 291-300, 1998.

[11] P. de Reffye, E. Heuvelink, D. Barthélémy, and P.-H. Cournède, "Plant growth models," in Ecological Models. Vol. 4 of Encyclopedia of Ecology (5 volumes), S. Jorgensen and B. Fath, Eds. Elsevier (Oxford), 2008, pp. 2824-2837.

[12] C. Varlet-Grancher, G. Gosse, M. Chartier, H. Sinoquet, R. Bonhomme, and J.-M. Allirand, "Mise au point : rayonnement solaire absorbe ou intercepte par un couvert végétal," Agronomie, vol. 9, pp. 419-439, 1989.

[13] S. Lemaire, F. Maupas, P.-H. Cournède, and P. de Reffye, "A morphogenetic crop model for sugar-beet (beta vulgaris l.)." in International Symposium on Crop Modeling and Decision Support: ISCMDS 2008, April 19-22, 2008, Nanjing, China, 2008.

[14] H. Yan, M. Kang, P. De Reffye, and M. Dingkuhn, "A dynamic, architectural plant model simulating resourcedependent growth," Annals of Botany, vol. 93, pp. 591-602, 2004.

[15] V. Letort, "Multi-scale analysis of source-sink relationships in plant growth models for parameter identification. case of the greenlab model." Ph.D. dissertation, Ecole Centrale Paris, 2008 .
[16] J. Bertheloot, B. Andrieu, F. Fournier, and P. Martre, "A process-based model to simulate nitrogen distribution in wheat (triticum aestivum) during grain-filling," Functional Plant Biology, vol. 35, pp. 781-796, 2008.

[17] J. Lizaso, W. Batchelor, and M. Westgate, "A leaf area model to simulate cultivar-specific expansion and senescence of maize leaves," Field Crops Research, vol. 80, 2003.

[18] C. Baker, C. Paul, and D. Wille, "Issues in the numerical solution of evolutionary delay differential equations," University of Manchester, Department of Mathematics, Numerical Analysis 248, 1999. 